\title{
PARCOURS MEMORIELS AMERINDIENS ET GEOPOETIQUE DES CONFINS AMERINDIAN MEMORIAL ITINERARIES AND GEOPOETICS OF THE CONFINES
}

\author{
Rita Olivieri-Godet ${ }^{1}$
}

Resume : Il sagit d'interroger les figurations des rapports des Amérindiens au continent américain dans des textes littéraires qui ont recours à la mémoire pour mettre en scène des "territoires lointains " et leurs populations autochtones: les territoires nordiques du Canada (Chants polaires de Jean Morisset, 2002) ; la forêt amazonienne (Yuxin-Alma de Ana Miranda, 2009); la Terre de Feu (Luna roja de Leopoldo Brizuela, 2002). De l'extrême-Nord à l'extrêmeSud du continent américain, en passant par la forêt amazonienne, c'est la construction imaginaire de la conception de "confins", renvoyant à la dichotomie entre civilisation et terres sauvages, qu'il nous intéresse d'examiner tout en soulignant les modalités spécifiques d'appropriation des éléments d'une mythologie de l'espace américain mises en place par les différents textes littéraires. Mots-cles: Mémoire, Amérindiens, Confins, Espace américain

Abstract: The representations of American Indians relationship to the American continent are investigated in literary texts that use memory to stage the "distant lands" and their indigenous peoples: the northern territories of Canada (Chants Polaires by Jean Morisset, 2002); the Amazonian forest (Yuxin-Alma by Ana Miranda, 2009); Tierra del Fuego (Luna roja by Leopoldo Brizuela, 2002). From the far north to the far south of the American continent through the Amazon forest, we are interested in the imaginary construction of the conception of "confines", referring to the dichotomy between civilization and wilderness, while emphasizing the specific modalities of appropriation of elements of a mythology of the American space implemented by the various literary texts. Keywords: Memory, Amerindians, Confines, American space

Ce travail se propose d'interroger les figurations des rapports humains aux confins du continent américain au sein de textes littéraires qui mettent

1 Professora Doutora da Université Rennes 2 - ERIMIT / Institut Universitaire de France: rgodet@9online.fr 
en scène des " territoires lointains » habités, ou ayant été habités, par des Amérindiens: les territoires nordiques du Canada (Chants polaires de Jean Morisset, 2002) ; la forêt amazonienne (Yuxin-Alma de Ana Miranda, 2009) ; la Terre de Feu (Luna roja de Leopoldo Brizuela, 2002). De l'extrême-Nord à l'extrême-Sud du continent américain, en passant par la forêt amazonienne, c'est la construction imaginaire de la conception de " confins »- appellation souvent utilisée pour désigner des terres «inexplorées», des terra incognita, renvoyant à la dichotomie entre civilisation et terres sauvages - qu'il nous intéresse d'examiner tout en soulignant les modalités spécifiques d'appropriation des éléments d'une mythologie de l'espace américain mises en place par les différents textes littéraires : la façon dont ils « inventent » le paysage ; leur " potentiel d'évocation iconique » (Philippe Descola) ; le statut métatextuel du paysage seront au centre de notre analyse.

La construction de l'espace littéraire est ici envisagée dans le sens de représentation (espace perçu) et de production de sens. Du topos au logos (AINSA: 2006: 9), comment les poèmes de l'écrivain-géographe-voyageur québécois, le roman de lécrivaine brésilienne et le récit de l'écrivain argentin fondent-ils des rapports à ces lieux emblématiques de l'espace ? Comment ces textes littéraires évoquent-ils la mémoire des rapports entre territoire et peuples amérindiens? Notre réflexion tient compte de cette capacité que certains textes littéraires manifestent à réinventer des mécanismes qui éveillent des mémoires ancestrales perdues, comme bien le souligne Zilá Bernd (BERND: 2013: 49).

\section{L’imaginaire des « confins " et les Amérindiens}

L'imaginaire qui s'est élaboré au fil du temps sur ces territoires considérés comme des " confins", met l'accent sur leur éloignement et sur leur isolement. Caractérisés comme des régions sauvages, ces territoires ne peuvent pas être dissociés de la représentation des peuples premiers qui les habitent en tant qu'instances d'altérité (PATTERSON: 2004) : les Indiens de l'Amazonie, les Inuit du Grand Nord canadien, les Onas et les Yaganes de la Terre de Feu, ces deux derniers ayant aujourd'hui disparu.

$\mathrm{Au}$ mot «confins » est associée l'idée de limite extrême d'un territoire. $\mathrm{Du}$ point de vue de l'histoire du continent américain, l'imaginaire des confins est en rapport avec le processus de démarcation territoriale qui débute avec la colonisation et sétend jusquà la période de la formation des 
nations. Ainsi, tout au long des siècles, on voit sériger un espace rationnel, limité, habité, "civilisé », l'espace de la ville, qui est aussi celui où siège le pouvoir institutionnel, en opposition à un espace périphérique, marginal, immense, désert, sauvage, qu'il faudra dompter, ainsi que les peuples qui y habitent, au nom de l'intérêt de la nation.

Comme le fait remarquer Claude Bourguignon (2010), la construction des espaces nationaux dans les Amériques sest réalisée par rapport au projet d'urbanisation. La vision que les premiers Européens ont projetée sur le " Nouveau Monde », opposant le "monde civilisé » au "monde sauvage », qu'elle soit euphorique ou dysphorique, est transplantée à l'intérieur des territoires qui se sont constitués en tant que nations. Tout l'enjeu actuel consiste non seulement à démasquer les bases sur lesquelles ces nations se sont formées mais aussi à interroger le processus de construction de leurs frontières internes et externes et, ce faisant, à s'ouvrir à une pensée sur le continent américain. Les cultures autochtones se trouvent ainsi au centre de ce questionnement sur l'identité continentale américaine du fait même de leur caractère transnational et de leur inscription dans la pré-histoire des Amériques.

Les ouvrages qui nous occupent s'inscrivent dans cette mouvance. Ils se livrent à l'édification d'un imaginaire qui se réapproprie les références culturelles des peuples premiers et leurs formes de rapport au monde pour inaugurer de nouveaux questionnements identitaires. Au-delà du présent historique, dans lequel les peuples premiers essayent de survivre malgré l'Occident qui les rattrape, Chants polaires, de Jean Morisset, Yuxin, de Ana Miranda, Luna roja de Leopoldo Brizuela instaurent un ordre symbolique basé sur la représentation d'un espace lointain et illimité en phase avec une dimension temporelle de très longue durée qui recule jusquaux temps immémoriaux. Dans leur effort de s'approprier la vision du monde des Inuit du Grand Nord canadien, d'un peuple indien de l'Amazonie ou celle des peuples indigènes de la Terre de Feu, ces auteurs témoignent de la résistance des peuples premiers, éveillent leur mémoire ancestrale, font appel à une étendue in illo tempore et suscitent une interrogation métaphysique sur lêtre humain et son rapport au monde.

\section{La mémoire invincible : Chants polaires de Jean Morisset}

Du recueil de poèmes intitulé Chants polaires de Jean Morisset émerge une voix poétique qui cherche à fusionner avec la nature physique et humaine pour évoquer l'expérience unique de ce territoire. La voix du sujet poétique, dans la poésie de Morisset, surgit de l'interaction du vivre et 
de l'écrire. Sa poésie est nourrie de l'expérience acquise dans le parcours nomade du poète à travers le continent américain - lui qui a navigué d'arctique en tropique "sans perdre ni sa coque ni son sillage", comme le vieux glacier de son poème (MORISSET: 2002 : 76-77).

La puissance créatrice de ses chants vient d'une parole poétique en quête ontologique qui inaugure une vision de l'intérieur tout en promouvant une sorte de «lecture cosmopoétique du monde " (WHITE: 1987: 357). D’un certain point de vue, les poèmes de Jean Morisset sont une invitation à l'expérience du Divers, pour reprendre l'expression de Victor Segalen (SEGALEN: 1978: 41) : ils imposent un déplacement spatial en évoquant l'espace lointain, méconnu et emblématique du Grand Nord canadien ; ils célèbrent l'altérité anthropologique; ils suggèrent, par l'hybridisme du langage qui incorpore au français des mots anglais et des mots inuktitut/inoutitout, des passages entre les mondes inuit et occidental. La parole poétique de Jean Morisset pousse cette expérience du divers encore plus loin, lorsquà travers les chants que le poète exécute, à l'unisson avec les éléments de la nature, il investit l'expérience ancestrale des Inughuit - les Inuit, les Esquimaux polaires :

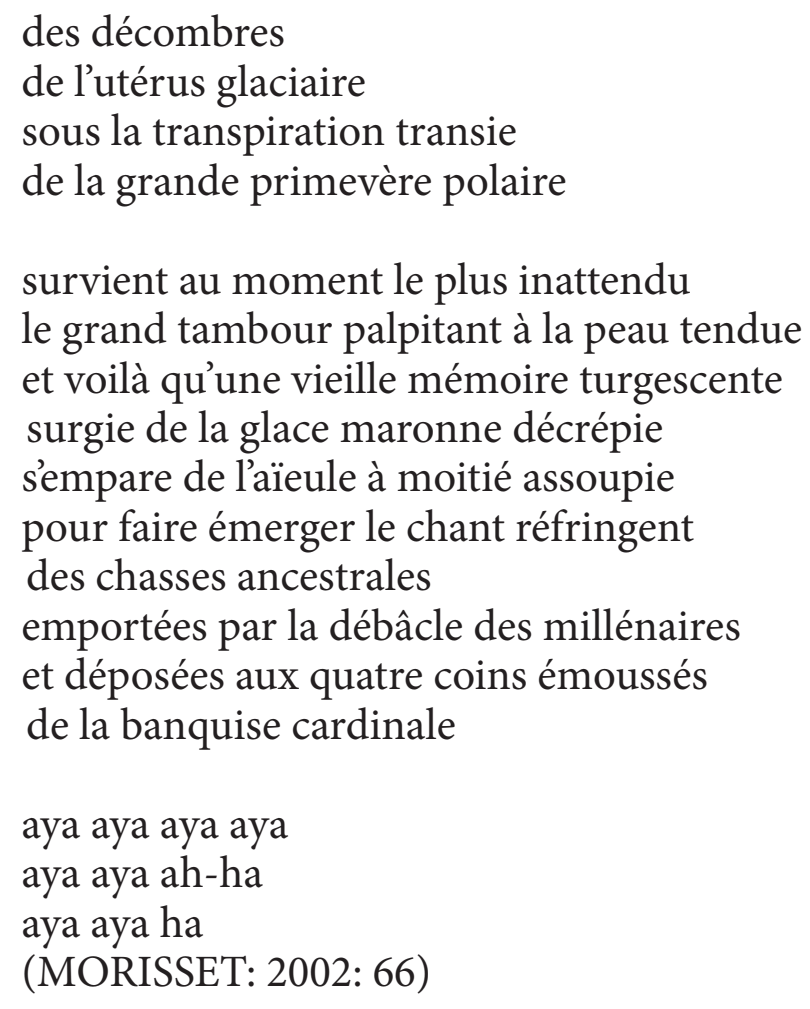

Dans ce poème, le choix du champ sémantique oppose des images d'un monde en voie de disparition à l'irruption d'un chant, qui puise sa force dans la nature, pour évoquer la mémoire ancestrale de la chasse, activité 
structurante du mode de vie de tout un peuple. La figure de l'aïeule renvoie au savoir transmis de génération en génération par les personnes âgées qui jouent, au sein des communautés des peuples premiers, le rôle de gardien de leur mémoire. Le symbolisme du renouvellement suggère des liens secrets qui sétablissent entre la résurgence du printemps et lémergence de ce chant de résistance qui nous transporte vers un passé où les Esquimaux étaient les maîtres de leur environnement. Le chant sélève contre la déchéance de ce peuple et la perte de ses références culturelles. Lart accomplit ainsi la fonction de maintenir éveillée la mémoire d'une communauté dont la vie s'est radicalement transformée, dans un intervalle de temps très court.

Cette dimension mémorielle qui traverse les poèmes des Chants polaires est ancrée dans une « faim archaïque » qui souvre à la mémoire d'un héritage millénaire, à une forme de connaissance élaborée et partagée par la communauté qui donnait sens à son existence et qui fait défaut dans le présent.

La transformation accélérée que l'Occident a imposée aux Esquimaux polaires, a été dénoncée par Jean Malaurie, ethno-historien et écrivain, spécialiste renommé de la géographie polaire. Dans un livre sur les Inuit de Thulé, au Nord du Groenland, l'auteur, qui les a fréquenté lors de ses expéditions, a pu témoigner de la transformation subie par cette population dans la deuxième moitié du XXe siècle : «De lâge du harpon et du phoque à l'âge du cosmos, en quarante-cinq ans : quel destin et quel défi ! » (MALAURIE: 1999:1: 292). Dans un autre ouvrage consacré aux chasseurs de morses et de caribous de l'Arctique central canadien, Malaurie témoigne de la situation des Netsilingmiut (Péninsule de Boothia), en 1961 : pauvreté, famine, manque de caribou dans ce territoire victime de la politique « indigène » avec sa mission " civilisatrice » implantée dans le Nord canadien. ${ }^{2}$

Le projet de Morisset fait écho, dans la poésie, au travail de Malaurie, dans la mesure où le poète cherche à éveiller une mémoire géographique de dimension archéologique, en fouillant les éléments d'un pay-

2 Jean Malaurie s'interroge sur la capacité de survie de certains groupes Inuit du Nord du Canada (Péninsule de Melville) qui doivent faire face au manque du caribou dans leurs territoires. Il dénonce également la mainmise occidentale sur ce territoire, comme c'est le cas pour l'usage belliqueux qui en est fait dans la période de la guerre froide : « Mais la vie est absente. Je ne vois aucune de ces bandes de caribous ou de bœufs musqués qui, il y a trente ans, étaient encore si nombreuses. Comment les Inuit - les Esquimaux Caribous, les plus archaïsants des Inuit-, liés exclusivement à la chasse continentale, survivent-ils ? Des groupes entiers de Naskapis, Indiens du Québec, disparurent en 1910 quand les hardes de caribous manquèrent. Nous survolons une base-relais de la Dew Line. Travail babylonien, entrepris en pleine guerre froide (1951), pour se prémunir contre une attaque surprise de Moscou et pouvoir riposter nucléairement à partir de Thulé ou des sous-marins lance-missiles en station secrète sous la glace de l'Océan glacial. » (Jean Malaurie, Hummoks 1, livre 2, p.82-83).

Organon, Porto Alegre, v. 29, n. 57, p. 61-76, jul/dez. 2014. 
sage archéen, précambrien, de cette terre première, pour évoquer l'aube de l'histoire, comme en témoignent les innombrables métaphores, auxquelles il a recours, renvoyant à l'enfance du monde. Assise sur une symbolique spatio-temporelle archéologique, la transposition de l'écriture géographique sur le terrain de la poésie, réalisée par cette parole poétique, dénote le désir de reterritorialiser l'imaginaire sur le « Nouveau Monde ». Face à l'ampleur de la tâche à laquelle le sujet poétique est confronté, celui-ci, en " chasseur-poète » (MORISSET: 2002: 64), hésite mais il ne renonce pas à " rescaper » ses "mots mouillés », au cours du voyage erratique que sa poésie inaugure. Le symbolisme cosmique explore l’opacité des signes. Malgré léternel retour des cycles temporels, lêtre humain n'est pas à même de les comprendre, incapable qu'il est de percer le secret de la vie. Les photographies qui interagissent avec le texte poétique rendent encore plus intense l'impuissance de lespèce humaine à dévoiler les mystères de la vie. Face à l'extrême beauté de ces immenses blocs de glaciers, témoins muets de la continuité de la vie, lénigme demeure entière pour celui qui contemple les nuances de blanc reproduites à l'infini.

\section{L'espace-temps amazonien dans Yuxin d'Ana Miranda}

La perspective temporelle adoptée par le récit d'Yuxin d'Ana Miranda est également celle de l'inscription dans un temps de longue durée qui se construit à partir d'une superposition de temporalités. Le roman s'applique à sonder les enjeux du présent en le mettant en rapport avec le temps immémorial projetant une visée de la trajectoire ancestrale et mythique de l'espace-temps amazonien.

Dans Yuxin, le temps présent est celui du début du XXe siècle lorsque les peuples amérindiens se voient confrontés à un flux migratoire important de travailleurs brésiliens attirés par la première explosion de la production du latex dans la région amazonienne. Le récit élabore une sorte d'ethnographie poétique du quotidien des Indiens, principalement inspirée de la culture des Kaxinawa. L'espace référent est celui de la frontière de l'actuel état de l'Acre avec le Pérou, région de grands fleuves et de frontières poreuses, habitée par des peuples d'une même ethnie qui se retrouvent des deux côtés de la ligne frontalière. Le choix fait par le roman pour appréhender cet espace physique et humain est délibérément poétique, laissant au lecteur la tâche de reconstituer les données historiques, sociologiques, anthropologiques à partir des indices éparpillés dans le récit. De même que 
pour les poèmes de Jean Morisset, avec lesquels l'auteur partage la perspective géo-poétique de l'espace, les rapports au temps et au lieu sont investis par les sensations, pour faire éclore une dimension affective de l'espace.

Par la voix de la narratrice-protagoniste Yuxin, dont le nom en langue kaxinawa signifie (approximativement) "âme », le lecteur pénètre dans un mystérieux univers orienté par son regard. Dès lors, il est submergé par une sensation d'étrangeté face à l'opacité des référents culturels, sensation que le rythme incantatoire du langage et la langue hybride auxquels le récit a recours - le portugais parsemé de mots kaxinawa et des onomatopées qui font écho aux éléments de la forêt - ne font que renforcer. Une lecture des premières lignes du chapitre qui ouvre le roman, intitulé « kene, bordado », nous transporte d'emblée dans cet univers lyrique que le langage poétique inaugure :

A pata da onça e aqui olho de periquito... bordar, bordar... Xumani está demorando tanto, quando ele voltar, amanhã, não vou contar nada, se eu contar, Xumani ciumento vai querer flechar as almas, matar as almas, quem pode as almas matar? Bordar bordar... hutu, hutu, hutu hutu...aprendi o bordado kene em dia de lua nova... bordar...bordar... achei aquele couro de cobra atrás do tear, minha avó me levou mata dentro para eu saudar Yube e aprender o bordado kene, minha avó ensinou as cantigas, aregrate mariasonte, mariasonte bonitito... ela sabia essas cantigas, a avó da avó sabia, a avó da avó da avó, minha mãe sabe... bonitito bonitito yare...tiriri tiriri tiriri tiriri wê...hutu, hutu, hutu, hutu... (MIRANDA: 2009, p. 17)

L'intrigue, très simple par ailleurs, met en scène la souffrance de la narratrice-personnage qui doit faire face à l'absence de son mari, Xumani. Celui-ci s'est vu obligé de quitter son village pour ne pas se faire tuer par ses ennemis appartenant à une autre tribu indienne. Tout comme Pénélope, Yuxin passe son temps à broder dans l'attente du retour de son aimé. Cette référence intertextuelle au récit d'Homère permet de mettre en parallèle lacte de broder et le tissage du fil du discours discontinu du personnage sur sa vie. Au-delà d'une simple référence intertextuelle au récit d'Homère, voire même d'une allusion auto-référentielle à la façon dont le récit se construit, en entrelaçant des éléments hérités des cultures amérindiennes et de la tradition littéraire brésilienne (dont Macunaíma et Maíra sont les exemples les plus évidents), l'activité du tissage fait partie de la tradition culturelle des Kaxinawa. ${ }^{3}$ La prise en compte de cette donnée du réel met

3 Voir Philippe Erikson, op. cit. p. 245.

Organon, Porto Alegre, v. 29, n. 57, p. 61-76, jul/dez. 2014. 
en relief le rôle de la mémoire généalogique dans la transmission de l'expérience cumulée au fil du temps. Tout comme dans le poème de Morisset, c'est la figure de l'aïeule qui est la gardienne de la mémoire ancestrale. C'est par l'intermédiaire de Mananan, son aïeule, que Yuxin hérite de l'histoire, des mythes et du savoir-faire de son peuple. La mémoire de longue durée est inscrite dans le récit qui ne cesse de renvoyer à cette forme de transmission de connaissance qui sétale dans le temps, par le biais d'un refrain construit sur le principe de la répétition des mots pour simuler le recul dans le temps: " os avós dos avós dos avós dos avós nos contaram » (MIRANDA: 2009: 126).

Ce procédé renvoie également à la dimension mythique du temps qui évoque les légendes et les croyances sur l'origine du monde. Celles-ci structurent la pensée animiste de ce peuple ainsi que sa vie sociale. La mémoire de longue durée instaure des liens pérennes entre la population et son territoire. Elle nous parle du désir de réinvestir cet espace, bouleversé par la dispute pour sa possession après le boom du latex. Ces éléments du contexte historique, que le récit explore à travers la reprise de l'opposition des marques temporelles " antes " (avant) versus " agora » (maintenant), justifient une construction imaginaire basée sur l'opposition temporelle entre un temps de plénitude révolu et un présent associé au manque.

Le présent est celui de la déterritorialisation, de la soumission à un ordre institutionnel religieux, de l'élargissement des conflits interethniques à des tribus qui se trouvent du côté du Pérou - les Takanawa -, de l'exposition à la violence des seringueiros et à celle de Brésiliens qui veulent tirer profit de la situation de fragilité dans laquelle se trouve la tribu de Yuxin. Le roman met en scène les modifications importantes de leur milieu de vie. Il fait allusion aux relations interethniques entre les tribus amérindiennes ainsi quaux relations qui caractérisent les groupes locaux vis-à-vis du monde occidental, dans ce territoire frontalier entre le Brésil et le Pérou. Il tire profit de cet espace frontalier dans lequel on assiste à l'exacerbation de la confrontation d'intérêts, en même temps que les différences culturelles semblent vouées à se confondre au fur et à mesure que les contacts et les échanges deviennent plus intenses.

Par la représentation de l'immensité de la forêt amazonienne, le roman rappelle que l'Amazonie dépasse les frontières nationales qui ont séparé des peuples appartenant à la même ethnie et à la même famille linguistique Pano (les Kaxinawa du Brésil et les Takanawa du Pérou). La mémoire de la forêt amazonienne est constituée par des affinités identitaires et culturelles que les peuples premiers de cette région frontalière, située entre les fleuves 
Purus et Juruá, se sont partagées tout au long des millénaires, malgré les différences et les conflits qui les ont opposés. Le contact avec l'Occident projette ces peuples dans un avenir incertain oscillant entre menace d'extinction de leurs références mémorielles ou culturelles et transformation de ces références par des passages interculturels ou transculturels. L’espace frontalier est aussi bien celui de la barrière qui circonscrit une limite que celui du passage, caractéristique de la fluidité des contacts.

La représentation de l'infini spatial d'un territoire qui est aussi perçu comme labyrinthique, un "emaranhado de mata e cipós ", (MIRANDA: 2009: 77) liée à la temporalité est propice au questionnement des rapports de lêtre humain au monde. A partir des singularités de son vécu spatiotemporel, Yuxin s'interroge sur l'énigme et les mystères de l'existence. Le rapport que le personnage établit au lieu et au temps souvre à une approche ontologique que l'on peut rapprocher de celle des poèmes de Morisset.

\section{La flamme de la mémoire : Luna roja de Leopoldo Brizuela}

Lélaboration du récit de Luna roja, centré sur la figure du foguista, explore la métaphore du gardien du feu. Au sein de la communauté des yaganes, le foguista avait pour tâche de transporter le feu dans le canoë, de l'alimenter et de le maintenir allumé, lors de la navigation dans les canaux, dans un milieu climatique extrême : "El mar de la Tierra del Fuego es el más peligroso de este mundo. En las tormentas, sólo el foguista parecía conservar la esperanza ». (BRIZUELA: 2002: 257). On peut lire Luna roja comme une allégorie du travail de l'écrivain, celui qui cherche à appréhender et à maintenir vivante la flamme de l'existence, celui qui garde la flamme qui illumine la mémoire, l'écriture étant, comme le feu, investie du symbole de la renaissance et de la permanence. Selon la signification que le texte confère au mot foguista, dans la langue yagana, celui-ci signifierait " gardien de la lune de la mer ", la petite lumière du feu qui résiste aux tempêtes et qui signale le canoë qui transporte les survivants. Par le recours à la métaphore du feu, il s'agit également, pour Brizuela, d'inscrire la temporalité dans une très longue durée, procédé qu'il partage avec les textes de Morisset et de Miranda.

Le sous-titre du récit Luna roja - «Apuntes sobre el oficio del foguista en las tribus canoeras de la Tierra del Fuego » laisse supposer que le lecteur aura affaire à une étude ethnographique sur les anciens habitants, aujourd'hui disparus, des confins sud du continent américain: les Yaganes (ou 
Yámanas et Alacufes) peuples de la mer, pêcheurs ; los Onas (ou Selknam) peuples de la montagne, chasseurs. Néanmoins, ce que l'auteur élabore, en effet, c'est une parodie d'études ethnographiques, de récits de voyage et de témoignages de missionnaires, navigateurs, voyageurs, explorateurs, colons et indigènes. Le croisement de tous ces discours constituera son tissu narratif qui interrogera la construction mythique sur cette région.

Le principe de construction du récit est basé sur une figuration qui explore le potentiel d'évocation iconique (DESCOLA) de la Terre de Feu et de ses habitants, en sondant des indices qui produisent l'image et qui demeurent actifs en celle-ci. C'est par des signes, des pistes, des indices que l'on pénètre dans la complexité de cet univers : réécriture de l'histoire ; inscriptions de mythes, de chansons, de rituels, de poèmes ; reprise, détournement, invention de témoignages. L'écrivain s'engage ainsi dans une construction imaginaire labyrinthique pour évoquer le quotidien du " foguista » dans un paysage inhospitalier, marqué par l'océan déchaîné et l'infini maritime, qu'il traverse en solitaire, au seuil des limites extrêmes de l'existence. Imitant la configuration de l'espace du prototype réel (DESCOLA) qu'il évoque - « labyrinthe d'îles et de canaux » ((BRIZUELA: 2002: 253) -, solitude d'un décor du bout de monde, le récit fait appel à des motifs qui confèrent au paysage un statut métatextuel.

A l'instar des Inuits, les Onas et les Yaganes de la Terre de Feu ont vu leur habitat et leurs cultures se transformer radicalement et abruptement dans un temps très court, l'espace de deux décennies à peine. Selon l'anthropologue Carlos Martínez Sarasola (1992 : 311-313), entre l'arrivée des premiers colons, en 1880 , et la première décennie du $\mathrm{XX}^{\mathrm{e}}$ siècle, ces peuples indigènes de l'Extrême sud du continent ont été victimes de massacres, épidémies, empoisonnements qui ont conduit à leur extermination. Pourtant, ces Indigènes avaient développé tout un savoir sur leur milieu qui leur ont permis de survivre dans des conditions climatiques extrêmes, pendant des milliers d'années : les Onas, dans les montagnes de la grande île de la Terre de Feu, se consacraient à la chasse tandis que les Yaganes, installés dans les zones du littoral, au sud et dans les Canales Fueguinos, s'adonnaient à la pêche et à la chasse maritime.

Ces deux peuples étaient considérés par des missionnaires et des scientifiques comme des représentants de cultures antithétiques. Néanmoins, tous deux s'auto-désignaient dans leurs langues respectives par le même nom : los que llegamos más lejos (nous qui sommes arrivés le plus loin). Cette forme d'identification commune renvoie, dans la logique singulière de leur perception, à une projection d'eux-mêmes comme un ensemble 
unitaire, malgré leurs différences, contrairement à la logique classificatoire adoptée par les occidentaux qui établit des frontières rigides. Le récit de Luna roja semble adhérer à une représentation relationnelle de l'altérité de ces peuples, comme on peut le lire dans la scène initiale reproduite ci-dessous :

Los onas, gente de las montañas de Tierra del Fuego, son cazadores y guerreros. Los yaganes, gente de las playas, son navegantes y pescadores.

Cada amanecer, desde las cumbres más altas, los onas ven poblarse los canales de una infinidad de canoas, colmadas com los frutos de la pesca nocturna. A proa de cada una hay un remero, a popa otro, y ambos reman con la recia mansedumbre del deber cumplido.

En medio de los dos, apenas se distingue la silueta del foguista. Encorvado, sopla y apantalla la última brasa en su brasero. (Brizuela: 2002: 249)

C'est par l'observation des signes de l'altérité des Yaganes que les Onas prennent conscience de leurs différences; leur regard vers l'autre construit un point de vue sur eux-mêmes. A travers cette projection miroir, le récit renforce l'idée d'une altérité complémentaire, d'une identification possible entre les deux peuples en renouant les liens qui les unissent de par leur origine - " hijos de una misma prehistoria" (p. 293) - et leur destin commun - "ya ambos pueblos se hallaban virtualmente extinguidos" (p. 290). En se reportant aux cérémonies rituelles et aux mythes des Onas et de Yaganes, le récit suggère une affinité relationnelle entre les deux peuples, en les faisant momentanément partager le territoire et lélément sacré de l'autre.

La figuration interactive des échanges culturels entre les Onas et les Yaganes, élaborée par le texte, est en phase avec une interprétation anthropologique de certaines sociétés amérindiennes qui voit, dans leur façon de penser leur rapport à l'autre, les signes d'une relation immanente avec l'altérité (VIVEIROS DE CASTRO: 2002: 220). Des sociétés dont le fondement même est le rapport à l'autre, qui se constituent à partir des échanges avec l'autre, au lieu de s'enfermer dans une identité substantielle. C'est ainsi que le récit de Luna roja multiplie les indices d'une expérience relationnelle de l'altérité entre les Onas et les Yaganes, dont la transformation des délimitations spatiales en frontières fluides, par l'interchangeabilité de la place que chacun des peuples occupe habituellement, est une des illustrations les plus expressives. Cet endroit "le plus éloigné », auquel les deux cultures font allusion, au-delà de la référence à l'expérience historique et mémoOrganon, Porto Alegre, v. 29, n. 57, p. 61-76, jul/dez. 2014. 
rielle partagée, fait écho également à l'expérience de l'extrême que le récit élabore en mimant la cartographie du territoire :

Onas y yaganes, ¿no recordarían de pronto, con la certeza deslumbrada de quien se mira por vez primera en un espejo, a aquellos remotísimos ancestros comunes que según se dice alguna vez partieron de Asia huyendo de alguna otra Tormenta Madre y que siguiendo a las golondrinas habían llegado a Alaska y cruzado Norteamérica, Centroamérica y Sudamérica hasta llegar aquí, sólo ansiosos de salvar su fuego, sólo deseosos de encontrar la isla en donde ninguna tormenta lo arriesgara? ¿No sería ese lugar más lejano, a la vez, aquel umbral de ambas memorias colectivas y este eterno final al borde de la tierra y de toda civilización, donde ya no se podía ir más allá?

«Tierra del Fuego », concluye Isáieva, « el lugar más lejano, tan al sur como puede irse en este mundo ; allí donde el misterio se volvía, en sí mismo, una respuesta, allí donde el silencio nos regala, como un árbol o una ballena, la poesía para siempre ». (Brizuela :2002:294)

Ainsi, la configuration géographique des confins renvoie à la frontière extrême entre la vie et la mort que le récit de Luna roja travaille dans deux directions : finitude et passage. A la première correspond le contexte de confrontation dans lequel s'inscrivent les rapports d'altérité entre les Amérindiens de la Terre de Feu et les Occidentaux. Le récit active la mémoire de l'histoire, celle de l'imposition de la logique de la conquête qui instaure l'invisibilité de l'autre et conduit à son anéantissement. Lautre façon de convoquer la frontière extrême est celle à laquelle la poésie adhère en faisant appel à des figures qui réactivent l'imaginaire de la perméabilité des extrêmes. D'un geste éthique et utopique, évoquant un rituel qui se perd dans la nuit des temps, l'écrivain-foguista renouvelle l'expérience-limite de l'écriture, en s'appropriant la réalité pour la réinvestir d'un sens, à la fois révélateur et énigmatique " para morir y renacer, morir y renacer, para salvarnos a nosotros todos » (BRIZUELA: 2002: 299).

\section{Parcours poétiques à travers les Amériques}

Le choix d'une représentation esthétique qui intègre la dimension ontologique de l'espace à sa dimension topologique enracine l'histoire et la mémoire dans un temps de très longue durée - la "pré-histoire " des 
Amériques - pour rompre avec une figuration qui fait coïncider l'origine du continent américain avec l'arrivée des Occidentaux. Le « Nouveau Monde » n’a jamais été un territoire vierge, sans histoire et sans mémoire. Il s'agit partant, pour nos écrivains, de faire interagir passé et présent en faisant appel à la mémoire du passé sans oublier les problèmes actuels suscités par une cohabitation qui saccélère et qui est en train de se dérouler sous nos yeux. Nous sommes tous concernés par ce présent, que nous soyons Inuit, Indiens de l'Amazonie, ou habitants des villes du continent américain. Les frontières sont en train de changer, où que nous nous situions : "Nous sommes tous contemporains »(AINSA: 2012: 75).

Ainsi, la représentation de ces territoires comme des confins est en train d'évoluer. Il n'y a plus de terres inconnues. Les " confins " ne sont plus isolés, mais se seraient-ils pour autant transformés en des «lieux de rencontre "? Ce n'est pas la lecture que les ouvrages qui nous occupent font du présent, plutôt marqué par des signes de déchéance, mais c'est ce que la dimension utopique de leurs œuvres projette. La question qui se pose est celle d'examiner dans quel sens bougent les frontières, devenues poreuses, de ces « confins »? Sur quelles bases pouvons-nous retracer le sens des Amériques? Comment agir pour favoriser l'entrecroisement et le dialogue entre les cultures, dans un espace américain qui est toujours en train de se (re)construire?

Pour nos auteurs, louverture à l'altérité des peuples premiers peut être une des réponses à ces questions. Leurs œuvres sont porteuses d'un espoir utopique, celui d'inclure l'héritage ancestral des peuples autochtones des Amériques dans l'expérience contemporaine du monde. Ils adoptent une perception poétique et sensorielle mettant en lumière une manière singulière d'habiter le lieu et le temps qui nous incite à " penser avec les sens " (MAFFESOLI: 2012: 32). Ce faisant, ils réintroduisent une dimension utopique profonde dans un présent qui souffre énormément du désenchantement du monde. Comme lécrit Gérard Bouchard, "la tâche présente appelle à réenchanter le monde, à inventer une transcendance ou son équivalent, à réconcilier mythe et raison, [...] à réhabiliter le mythe comme ressort de la pensée » (BOUCHARD: 2003:117).

\section{BIBLIOGRAPHIE}

AINSA, Fernando. Del topos al logos. Propuestas de géopoética. Iberoamericana/Vervuert: Madrid/Frankfurt, 2006. 
AINSA, Fernando. Palabras nómadas. Nueva cartografía de la pertenencia. Iberoamericana/Vervuert: Madrid/Frankfurt, 2012. ANDRADE, Mário. Macunaíma: o herói sem nenhum caráter. Edição crítica de Telê Porto Ancona Lopez (coordenadora). $2^{\mathrm{a}}$ ed. Madrid; Paris; México; Buenos Aires; São Paulo; Rio de Janeiro; Lima: ALLCA XX, 1996. BACHELARD, Gaston. La poétique de la rêverie. Paris : Quadrige/PUF, 1993. BARON, Christine. Littérature et géographie : lieux, espaces, paysages et écritures. Le partage des disciplines, LHT, Dossier, publié le 16 mai 2011[En ligne], URL : http://www.fabula.org/lht/8/index.php?id=221 . BERND, Zilá. Américanité et mobilités transculturelles. Québec : PUL, 2009. BERND Zilá. Por uma estética de vestígios memoriais. Belo Horizonte: Fino Traço Editora, 2013. BERND, Zilá, Dicionário de Figuras e mitos literários das Américas. UFRGS Editora/Tomo Editorial: Porto Alegre, 2007.

BOURGUIGNON, Claude. Stratégies romanesques et construction des identités nationales. Essai sur l'imaginaire post-colonial dans quatre fictions de la forêt. Thèse de doctorat, Université de Grenoble, novembre 2010, consultable sur le site http://tel.archives-ouvertes.fr/docs/00/58/05/61/PDF/_StratA_gies_ romanesques_.pdf

BOUCHARD, Gérard. Jeux et nœuds de mémoire : l'invention de la mémoire longue dans les nations du Nouveau Monde. In: ANDRES, Bernard ; BOUCHARD Gérard (sous la direction de), Mythes et sociétés des Amériques. Montréal: Québec Amérique, 2007, p. 315-348. BOUCHARD, Gérard, Genèse des nations et cultures du Nouveau Monde. Montréal: Boréal, 2000.

BOUCHARD, Gérard. Raison et contradiction: le mythe au secours de la pensée. Québec: Nota Bene/Cefan, 2003.

BRIZUELA, Leopoldo. Luna roja. Los que llegamos más lejos. Buenos Aires : Alfaguara, 2002, p. 245-307.

COTE, Jean-François; TREMBLAY, Emmanuelle. Le nouveau récit des frontières dans les Amériques. Québec : PUL, 2005.

DAHAN-GAIDA, Laurence. E. La géocritique au confluent du savoir et de l'imaginaire. Epistémocritique, Volume IX, automne 2011 consultable sur le site

DESCOLA, Philippe. Lécologie des autres. L'anthropologie et la question de la nature. Versailles : Editions Quae : 2011.

ESTEVES, Antonio R.. Inferno verde. In: BERND, Zilá, Dicionário de Figuras e mitos literários das Américas. UFRGS Editora/Tomo Editorial: Porto Alegre, 2007, p. 340-345. 
ERIKSON, Philippe. Uma singular pluralidade: a etno-história Pano. IN: CUNHA, Maria Manuela Carneiro da (org.). História dos indios do Brasil. São Paulo: Companhia das Letras/FAPESP, 1992, p 239-252. GODET, Rita-Olivieri. A alteridade ameríndia na ficção contemporânea das Américas. Brasil, Argentina, Quebec. Belo Horizonte: Fino Traço Editora, 2013.

LOUVEL, Liliane. Texte/Image. Images à lire, textes à voir. Rennes : Presses Universitaires de Rennes, 2002. LUCCHESI, Marco. Yuxin-Alma (présentation). In : Yuxin-Alma. São Paulo : Companhia das Letras, 2009.

MAFFESOLI, Michel. Homo eroticus. Des communions émotionnelles. Paris : CNRS Editions, 2012.

MALAURIE, Jean. Hummosks. Tome 1. Livre 1. De la pierre à l'homme, avec les Inuit de Thulé. Paris : Plon, 1999.

MALAURIE, Jean. Hummosks. Tome 1. Livre 2. Avec les chasseurs de morses et des caribous de l'Arctique central canadien. Paris : Plon, 1999. MIRANDA, Ana. Yuxin-Alma. São Paulo : Companhia das Letras, 2009. MORISSET, Jean. Chants Polaires. Liminaire de Nancy Huston. Leméac/ Actes Sud : Montréal/Arles, 2002.

MORISSET, Jean. Les chiens séntredévorent...Indiens, Blancs et Métis dans le Grand Nord canadien. Montréal : Mémoire d’encrier, 2009.

MORISSET, Jean. Récits de la terre première. Montréal : Leméac, 2000. MORISSET, Jean et WADDEL, Eric. Amériques. Montréal : Editions de l'Hexagone, 2000.

OTENIO, Marta, Grand Nord. BERND, Zilá, Dicionário de Figuras e mitos literários das Américas. UFRGS Editora/Tomo Editorial: Porto Alegre, 2007, p.306-311.

PAGEAUX, Daniel-Henri. De la géocritique à la géosymbolique. Regards sur un champ interdisciplinaire : littérature générale et comparée et géographie. In : WESTPHAL, Bertrand (sous la direction de). La géocritique mode d'emploi. Limoges: PULIM, 2000, p. 125160.

PATERSON, Janet M.. Figures de l'autre dans le roman québécois. Québec : Editions Nota Bene, 2004.

RIBEIRO, Darcy, Maíra: um romance dos índios e da Amazônia. 21 a edição. Rio de Janeiro: Record, 2007 [1976].

ROSA, João Guimarães. Meu tio o Iauaretê. In: Estas estórias. Rio de Janeiro: José Olympio, 1969, p. 126-159.

ROSEMBERG, Muriel. Contribution à une réflexion géographique sur les représentations et l'espace. Géocarrefour, Vol.78/1/2003, 71-77.

http://geocarrefour.revues.org/130

Organon, Porto Alegre, v. 29, n. 57, p. 61-76, jul/dez. 2014. 
SEGALEN, Victor, Essai sur l'exotisme. Paris : Fata Morgana, 1978. WESTPHAL, Bertrand (sous la direction de). La géocritique mode d'emploi. Limoges : PULIM, 2000.

WHITE, Kenneth, L'esprit nomade. Paris : Editions Grasset et Fasquelle, 1987.

Recebido em: 26/06/2014. Aceito em: 05/08/2014. 